\title{
Changes in response properties of nociceptors and dorsal horn neurons in a murine model of cancer pain
}

\author{
Donald A. Simone*, Sergey G. Khasabov, David M. Cain, Darryl T. Hamamoto, and \\ Iryna A. Khasabova
}

Department of Diagnostic and Biological Sciences, School of Dentistry, University of Minnesota simon003@umn.edu

Pain is a common symptom in patients with cancer. It is estimated that over half of patients with cancer experience pain, up to $90 \%$ of patients in advanced disease stages experience pain, and metastasis to bone often causes severe pain. Although the prevalence and severity of cancer pain differs among the various types of cancer, $60 \%$ of individuals with primary or metastatic bone cancer suffer from severe pain. Severe cancer pain is most often treated with opioids, however, they do not always provide adequate analgesia and their use is limited by a variety of side effects.

Pain from cancer is unique and complex because it may be mediated by inflammation, neuropathy, and mediators released from the cancer cells, and is therefore difficult to manage. It is therefore important to gain a greater understanding of the mechanisms that contribute to cancer pain so that new targets for pain control can be identified. Animal models of cancer pain have been developed fairly recently and are providing information on the mechanisms that contribute to cancerrelated pain. Implantation of various types of tumor cells into or near bone in rodents produces ongoing nocifensive behaviors and hyperalgesia to mechanical, heat, and cold stimuli.

We were interested in identifying interactions between tumor and peripheral nerve, and neural encoding in the peripheral nervous system and in the spinal cord that contribute to cancer pain. We used a model in which fibrosarcoma cells are implanted into and around the calcaneus bone in the mouse. As the tumor grows, the tumorbearing paw develops hyperalgesia to mechanical, heat and cold stimuli that is maximal 10-14 days after implantation. Here we summarize electrophysiological and morphological changes that occur in primary afferent nerve fibers with tumor growth as well as changes in the encoding properties of nociceptive dorsal horn neurons that are likely to contribute to cancer pain. We also demonstrate that peripheral application of cannabinoids at the tumor site reduced hyperalgesia

Changes in the physiology and morphology of primary afferent nerve fibers during tumor growth Electrophysiological recordings were made from single Ад and C primary afferent fibers in the tibial nerve of mice with sarcoma cells implanted into and around the calcaneus bone. All receptive fields (RFs) were located on the plantar surface of the tumor-bearing hind paw. Approximately $30 \%$ of $\mathrm{C}$-fiber nociceptors exhibited spontaneous activity in (Cain et al., 2001). No changes were observed in their mechanical sensitivity using calibrated von Frey monofilaments, but mean heat response thresholds of C-fibers was lower in tumorbearing mice $\left(37.8 \pm 0.9^{\circ} \mathrm{C}\right)$ as compared to control mice $\left(40.3 \pm 0.4^{\circ} \mathrm{C}\right)$. Also, responses of $\mathrm{C}$ nociceptors to noxious cold were enhanced for stimulus temperatures lower than $10^{\circ} \mathrm{C}$. Interestingly, responses of A-fiber nociceptors evoked by mechanical and thermal stimuli were not altered during tumor growth. Thus, ongoing spontaneous activity and increased responses to heat and mechanical stimuli of C-fiber nociceptors are likely to contribute to spontaneous pain and to heat and cold hyperalgesia associated with this model of cancer pain. 
In addition to electrophysiological changes in C-fiber nociceptors, tumor development also produced morphological changes in nerve fibers. We obtained punch biopsies $(3 \mathrm{~mm})$ from plantar skin overlying the tumor. Epidermal nerve fibers (ENFs), which are unmyelinated nociceptive nerve fibers, were labeled using protein gene product 9.5 and epidermal innervation was quantified. It was found that early during tumor growth (approximately 10 days after implantation), there was a proliferation of ENFs as indicated by increased and complex branching of these fibers (Figure 1). However, as the tumor developed further, it produced neuropathy as indicated by a decrease in the number of labeled ENFs, This demonstrates
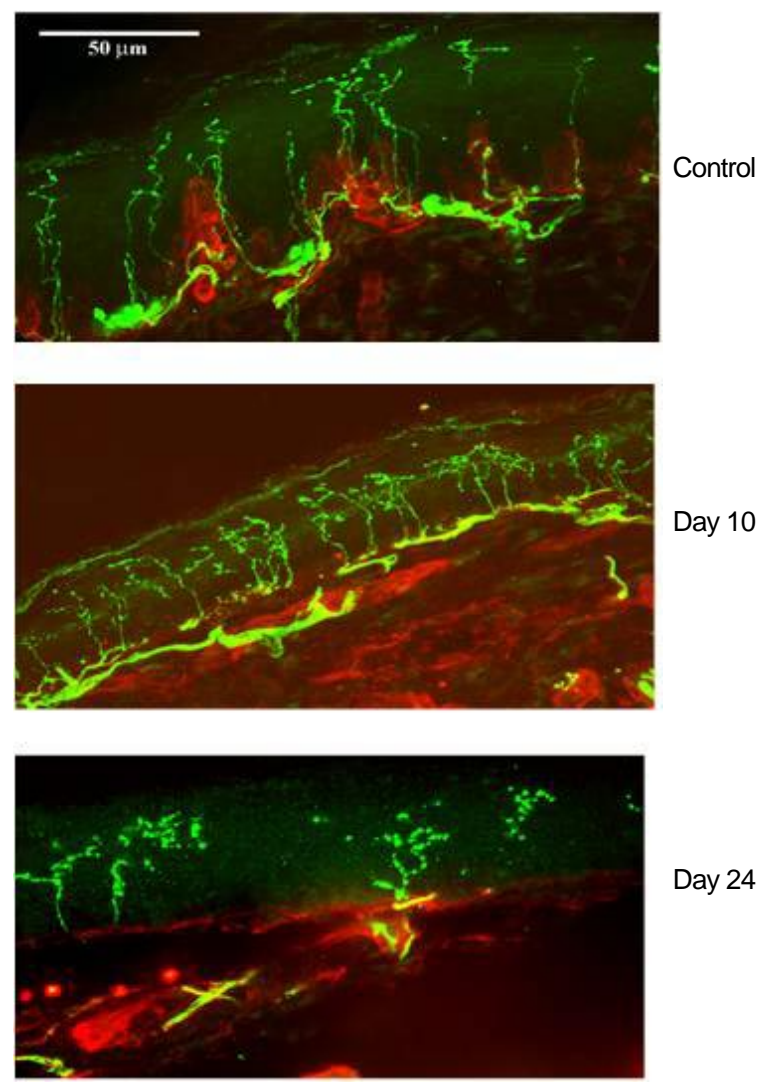

Day 24

Figure 1. Confocal images showing changes in epidermal innervation associated with tumor growth. Biopsies were obtained from the plantar surface of the tumor-bearing hind paw. Top: ENFs in a control mouse. Middle: Proliferation of ENFs at 10 days after tumor cell implantation. Bottom: Reduced number of ENFs at 24 days after tumor cell implantation. ENFs appear green that as the tumor grows, nerve fibers are injured and the associated pain and hyperalgesia is due in part to neuropathic mechanisms.

Response properties of dorsal horn neurons during tumor growth

Extracellular recordings were made from nociceptive dorsal horn neurons located in control and in tumor-bearing mice. All RFs were located on the plantar surface of the hind paw. Nociceptive neurons were classified functionally as wide dynamic range (WDR) or high threshold (HT) according to their responses evoked by mechanical stimuli of varying intensities.

Several differences in response properties were observed in tumor-bearing mice. First, a greater proportion (76\%) of WDR neurons exhibited ongoing activity in tumor-bearing mice as compared to controls (54\%) and their discharge frequency was approximately 3 times greater than that of control mice. Second, responses of WDR neurons to mechanical stimuli (brush, von Frey monofilaments, and pinching with serrated forceps) applied to the RF were greater as compared to those in control mice. Third, responses of WDR and HT neurons evoked by thermal stimuli were increased in tumor bearing mice (Figure 2).

\section{Effect of cannabinoids on tumor-evoked hyperalgesia}

Because of the limitations of prolonged opioid use for cancer pain, we investigated whether cannabinoids would reduce the hyperalgesia in our mouse model. Since peripheral antinociceptive effects of cannabinoids have been documented, we administered the nonselective cannabinoid receptor agonist, WIN 55,212-2, into the tumor-bearing paw. The frequency of withdrawal responses to mechanical stimuli (3.4 $\mathrm{mN}$ von Frey monofilament) applied to the plantar surface of the tumor-bearing paw was determined before and at various times after injection. As shown in Figure 3, WIN 55,212-2 reduced the frequency of withdrawal responses dose-de- 

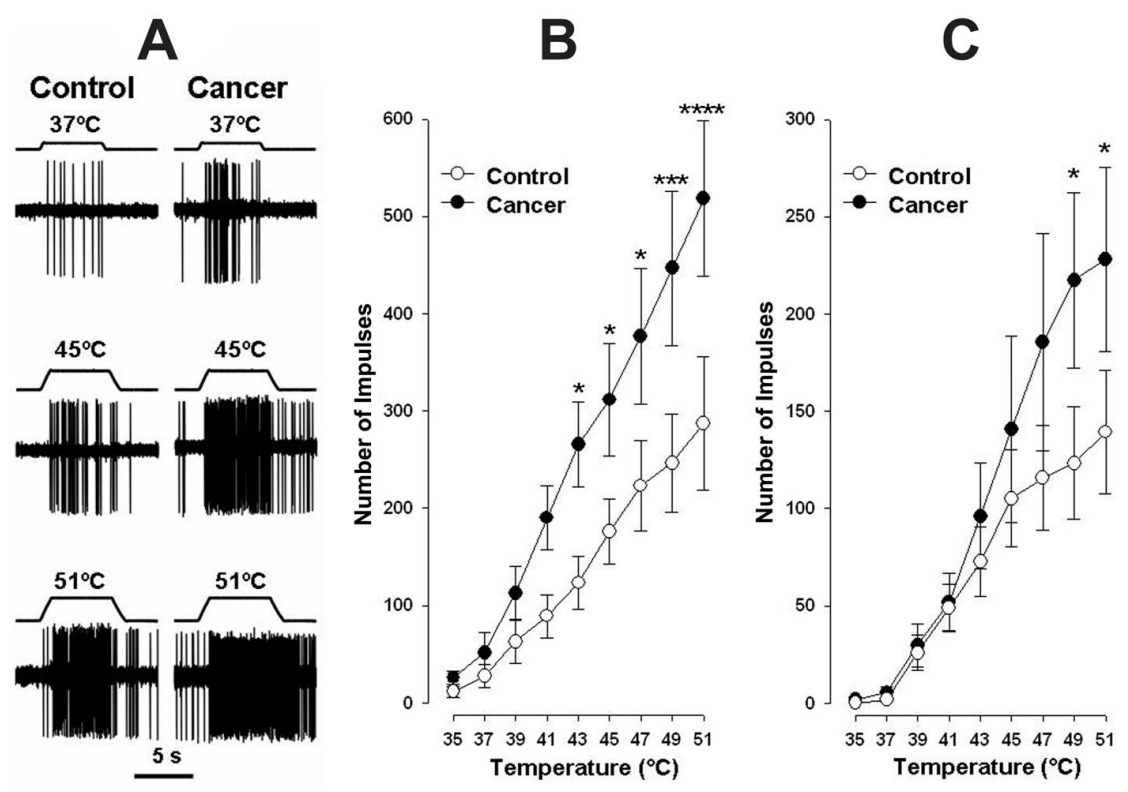

Figure 2. Effect of tumor growth on responses of nociceptive dorsal horn neurons to heat stimuli. A: Examples of the responses of individual WDR neurons evoked by $37^{\circ}, 45^{\circ}$ and $51^{\circ} \mathrm{C}$ from a control mouse and a tumor-bearing mouse. B: Heat stimuli evoked greater responses (mean number of impulses) from WDR neurons in tumor-bearing mice as compared to control mice. C: There were no differences in the mean $( \pm$ sem $)$ number of impulses from HT neurons evoked by the heat stimuli below $49^{\circ} \mathrm{C}$ between tumor-bearing and control mice. $* \mathrm{P}<0.05, * * * \mathrm{P}<0.005, * * * * \mathrm{P}<$ 0.001 for number of impulses between tumor-bearing and control mice at each temperature. From Khasabov et al., 2007

pendently. Additional studies using selective cannabinoid receptor antagonists indicated that this occurred through both $\mathrm{CB} 1$ and $\mathrm{CB} 2$ receptors. These studies suggest that cannabinoids, acting through peripheral mechanisms, may be beneficial in the management of cancer pain.

Implantation of sarcoma cells into and around the calcaneus bone produces robust hyperalgsia to mechanical, heat, and cold stimuli that is mediated by sensitization of C-

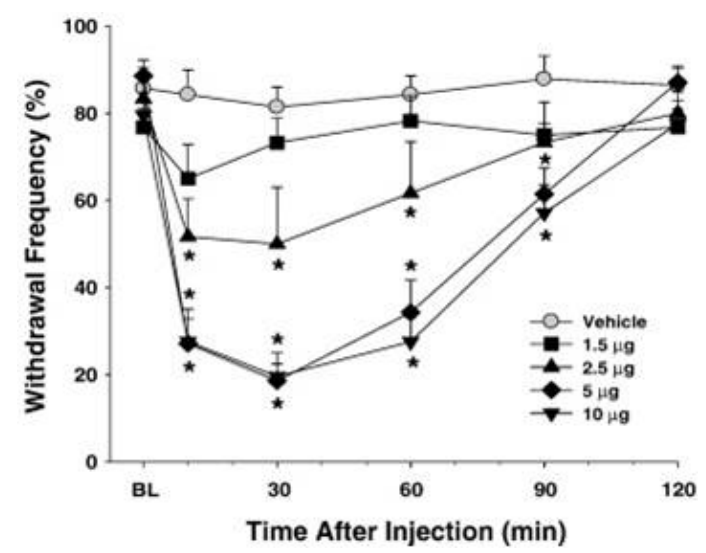

Figure 3. WIN 55,212-2 dose-dependently attenuates mechanical hyperalgesia. Local injection of $2.5,5$, or $10 \mathrm{mg}$ of WIN 55,212-2 into the tumor-bearing hindpaw reduced the mean paw withdrawal frequency evoked by a von Frey monofilament $(3.4 \mathrm{mN})$. indicates a significant difference from vehicle $(\mathrm{p} \leq 0.05)$. From Potenzieri et al., 2008 fiber nociceptors as well as central sensitization, particularly sensitization of WDR neurons. Some of these changes are unique to the cancer pain model and differ from those observed following inflammation alone, supporting the notion that separate mechanisms underlie cancer pain. Understanding the changes in the physiology and neurochemistry of the dorsal horn produced by tumor growth may provide new opportunities for management of cancer pain.

As we have shown, cannabinoids may be effective in treating cancer pain. Importantly, if cannabinoids are effective through peripheral mechanisms alone, this would avoid unwanted side effects associated with activation of cannabinoid receptors in the central nervous system. It has been shown that cannabinoids can decrease excitability or nociceptors. Thus, cannabinoids may decrease the ongoing activity and sensitization of nociceptors produced by tumor growth, thereby preventing the development of central sensitization and persistent pain.

These studies were supported by grants from the National Institutes of Health; DA011471 and CA091007. 